\title{
Efficient Buffering in Reliable Multicast Protocols
}

\author{
Oznur Ozkasap, Robbert van Renesse, Kenneth P. Birman, and Zhen Xiao \\ Dept. of Computer Science, Cornell University \\ 4118 Upson Hall, Ithaca, NY 14853 \\ ozkasap@bornova.ege.edu.tr, $\{$ rvr,ken, xiao\}@cs.cornell.edu
}

\begin{abstract}
Reliable multicast protocols provide all-or-none delivery to participants. Traditionally, such protocols suffer from large buffering requirements, as receivers have to buffer messages, and buffer sizes grow with the number of participants. In this paper, we describe an optimization that allows such protocols to reduce the amount of buffering drastically at the cost of a very small probability that all-or-none delivery is violated. We analyze this probability, and simulate an optimized version of an epidemic multicast protocol to validate the effectiveness of the optimization. We find that the buffering requirements are sub-constant, that is, the requirements shrink with group size, while the probability of all-or-none violation can be set to very small values.
\end{abstract}

\section{Introduction}

The aim of reliable multicast protocols is to provide all-or-none delivery of messages to all participants in a group 1 Informally, if any participant delivers the message, then eventually all participants should deliver the message. Since the sender may fail, processes buffer the messages that they receive in case a retransmission is necessary. Most existing reliable multicast protocols have all receivers buffer messages until it is known that the message has become stable (i.e., has been delivered to every participant).

In such systems, it is always the case that the amount of buffering on each participant grows with group size for a combination of the following reasons:

1. the time to accomplish stability increases;

2. the time to detect stability increases;

3. depending on the application, the combined rate of sending may increase.

As a result, these multicast protocols do not scale well.

\footnotetext{
${ }^{0}$ This work is supported in part by ARPA/ONR grant N00014-92-J-1866, ARPA/RADC grant F30602-96-1-0317, NSF grant EIA 97-03470, and the Turkish Research Foundation.

${ }^{1}$ We use here the distributed systems terminology for reliability [HT94, rather than the networking terminology which does not stipulate all-or-none delivery.
} 
In this paper, we investigate optimizing buffering by only buffering messages on a small subset of participants, while spreading the load of buffering over the entire membership. This way, each participant only requires a fraction of the buffering space used previously. Indeed, the amount of buffering space per participant decreases with group size.

On the negative side, we introduce a small, known probability that a message is not delivered to all members. For example, this may happen if the entire subset responsible for buffering the message crashes before the message is delivered everywhere. We believe that in many situations such small probabilities can be condoned. In fact, epidemic multicast protocols such as used in the Clearinghouse domain name service [ $\mathrm{DGH}^{+} 87$ ], Refdbms GLW94, Bayou $\left[\mathrm{PST}^{+} 97\right.$, and Cornell's bimodal multicast $\left[\mathrm{BHO}^{+} 99\right]$ already introduce such a small known probability. Because of this, we focus our attention on using our suggested optimization in such protocols.

Note that, using the terminology of [HT94, the resulting protocols are not reliable. Yet, we consider the robustness of these protocol better than protocols such as RMTP [LP96 or SRM [FJL ${ }^{+97}$ ], because the probability of message loss is known and therefore a certain known Quality of Service is provided (even if the original sender of a message fails). Our protocols are useful for life and mission-critical applications such as air traffic control, health monitoring, and stock exchanges, where a certain a priori known probability of message loss is acceptable $\left[\mathrm{BHO}^{+} 99\right]$. For such applications as money transfer, fully reliable all-or-none multicast or atomic transactions would be necessary, while for nonlife or mission-critical applications, such as tele-conferencing or distribution of software, protocols such as RMTP and SRM are sufficient.

We investigate techniques for choosing suitable subsets of participants for buffering messages, ways for locating where messages are buffered in case a retransmission is required, how these techniques improve memory requirements, and how they impact the reliability of the multicast protocols. We take into account message loss and dynamic group membership. For analysis we use both stochastics and simulation.

This paper is organized as follows. In Section 2, we describe the group membership model, as well as how reliable multicast protocols (particularly epidemic protocols) are structured. The buffer optimization technique is presented in detail, and analyzed stochastically, in Section 3. Section 4 describes how this technique may be incorporated into an existing multicast protocol. In Section [5], we weaken our assumptions and describe a technique to improve the reliability of the optimized protocol without sacrificing the scalability. Simulation results are presented in Section [6] Section [7describes related work, and Section 8 concludes.

\section{Model and Epidemic Protocols}

We consider a single group of processes or members. Each member is uniquely identified by its address. Each member has available to it an approximation of the entire membership in the form of a set of addresses. We do not require 
that the members agree on the membership, such that a scalable membership protocol such as vRMH98 suffices to provide this membership information. We consider a non-Byzantine fail-stop model of processes. As is customary, recovery of a process is modeled as a new process joining the membership.

The members can send or multicast messages among each other. There are two kinds of message loss: send omissions and receive omissions. In case of a send omission, no process receives the message. In case of a receive omission, only a corresponding receiver loses the message. Initially, we assume that receive omissions are independent from receiver to receiver and message to message, and occur with a small probability $P_{l o s s}$, and that there are no send omissions. We weaken these assumptions in Section 5.

The members run a reliable multicast protocol that aims to provide allor-none delivery of multicast messages, that is, to deliver each message to all processes that are up (not failed) at the time of sending. We do not require FIFO or total order on message delivery. All such protocols run in three phases:

1. an initial (unreliable) multicast phase attempts to reach as many members as possible;

2. a repair phase detects message loss and retransmits messages;

3. a garbage collection phase detects message stability and releases buffer space.

Most protocols use a combination of positive or negative acknowledgment messages for the last two phases. Epidemic multicast protocols accomplish the all-or-none guarantee with high probability by a technique called gossiping. Each member $p$ periodically chooses another member $q$ at random to send a gossip message to, which includes a report of what messages $p$ has delivered and/or buffered. (Every message that is buffered by a process has been delivered by that process, but not necessarily vice versa.) $q$ may update $p$ with messages that $q$ has buffered, but $p$ has not delivered. $q$ may also request from $p$ those messages that $p$ has buffered but $q$ has not delivered.

Garbage collection in epidemic protocols is accomplished by having members only maintain messages in their buffer for a limited time. In particular, members garbage collect a message after a time at which they can be sure, with a specific high probability, that the gossiping has disseminated all messages that were lost during the initial multicast. This time grows $O(\log n)$, where $n$ is the size of the membership as the corresponding member observes it $\left[\mathrm{BHO}^{+99}\right]$.

\section{Basic Optimization}

In this section, we describe the technique we use to buffer messages on only a subset of the membership. The subset has a desired constant size $C$. We say desired because, as we shall see, failures and other randomized effects cause messages to be buffered on more or fewer than $C$ members. The subset is not fixed, but randomized from message to message in order to spread the load of buffering evenly over the membership. 
We assume that each message is uniquely identified, for example by the tuple (source address, sequence number). Using a hash function $H$ : bitstring $\rightarrow$ $[0 \cdots 1]$, we hash tuples of the form 〈message identifier, member address $\rangle$ to numbers between 0 and 1 . This hash function has a certain fairness property, in that for a set of different inputs, the outputs should be unrelated. Cryptographic hashes are ideal, but too CPU-intensive. CRCs (cyclic redundancy checks) are cheap, but the output is too predictable for our purpose: when given the 32-bit big-endian numbers $0,1,2, \ldots$ as input, the output of CRC-16 is $0,256,512$, etc. We will describe a hash function that is cheap and appears fair, as well as why we require these properties, in Section 4

A member with address $A$ and a view of the membership of size $n$ buffers a message with identifier $M$ if and only if $H(\langle M, A\rangle) \times n<C$. We call a member that buffers $M$ the bufferer of $M$. If $H$ is fair, $n$ is correct, and there is no message loss, the expected number of bufferers for $M$ is $C$. Also, for a set of messages $M_{1}, M_{2}, \ldots$, the messages are buffered evenly over the membership.

If members agree on the membership, then any member can calculate for any message which members are the bufferers for this message. If members have slightly different memberships, it is possible that they disagree on the set of bufferers for a message, but not by much. In particular, the sets of bufferers calculated by different members will mostly overlap.

Also, if $C$ is chosen large enough, the probability that all bufferers fail to receive a message is small. We will now calculate this probability. For simplicity, we assume that every member agrees on the membership (and is therefore correct), and that this membership is of size $n$. We consider an initial multicast successful if it is received by all members, or if it is received by at least one bufferer (which can then satisfy retransmission requests). Thus, the probability of success is the sum of the following two independent probabilities:

$P_{1}$ : no members are bufferers, but they all received the initial multicast;

$P_{2}$ : there is at least one member that is a bufferer and that received the initial multicast.

$P_{1}$ is simple to calculate, as, by fairness of $H$, "being a bufferer" is an independent event (with probability $C / n$ ), as is message loss (with probability $\left.P_{\text {loss }}\right)$ :

$$
P_{1}=\left(\left(1-\frac{C}{n}\right) \cdot\left(1-P_{\text {loss }}\right)\right)^{n}
$$

$P_{2}$ can be calculated as follows:

$$
\begin{aligned}
P_{2} & =P(\exists \text { bufferer that receives } M) \\
& =1-P(\text { all processes are not bufferers or lose } M) \\
& =1-P(\text { a process is not a bufferer or loses } M)^{n} \\
& =1-\left(1-P(\text { a process is bufferer and receives } M)^{n}\right. \\
& =1-\left(1-\frac{C}{n} \cdot\left(1-P_{\text {loss }}\right)\right)^{n}
\end{aligned}
$$


The probability of failure $P_{\text {fail }}$ is then calculated as:

$$
P_{\text {fail }}=1-P_{1}-P_{2}=\left(1-\frac{C}{n} \cdot\left(1-P_{\text {loss }}\right)\right)^{n}-\left(\left(1-\frac{C}{n}\right) \cdot\left(1-P_{\text {loss }}\right)\right)^{n}
$$

Assuming $P_{\text {loss }}$ is constant (independent of $n$ ), it is easy to see that as $n$ grows, $P_{\text {fail }}$ tends to $\mathrm{e}^{-C \cdot\left(1-P_{\text {loss }}\right)}$. Thus, given the probability of receive omission, the probability of failure can be adjusted by setting $C$, independent of the size of the membership. $P_{\text {fail }}$ gets exponentially smaller when increasing $C$.

In many cases $P_{\text {loss }}$ is a function of group size, as it depends on the size and topology of the underlying network. For example, in a tree-shaped topology, messages have to travel over $O(\log n)$ links. If $P_{l l}$ is the individual link loss, then $P_{\text {loss }}=1-\left(1-P_{l l}\right)^{t}$, where $t$ is the average number of links that the message has to travel $(t$ grows $O(\log n))$. Worse yet, receive omissions are no longer independent from each other. Thus, setting $C$ in this case does depend on $n$. We discuss a solution to this problem in Section 5, and see how this affects the choice of $C$ in Section 6 .

\section{Implementation}

In this section, we discuss the design of the hash function $H$ that we use, how we integrate our optimization with an epidemic multicast protocol, and how this affects the buffering requirements of the protocol.

As mentioned, the hash function $H$ has to be fair and cheap. It has to be fair, so that the expected number of bufferers for a message is $C$, and so that the messages are buffered evenly over the membership. It has to be cheap, since it is calculated each time a message is received. Cryptographic hashes are typically fair, but they are not cheap. CRC checks are cheap, but not fair. We therefore had to design a new hash function.

Our hash function $H$ uses a table of 256 randomly chosen integers, called the shuffle table. The input to $H$ is a string of bytes, and the output is a number between 0 and 1 . The algorithm is:

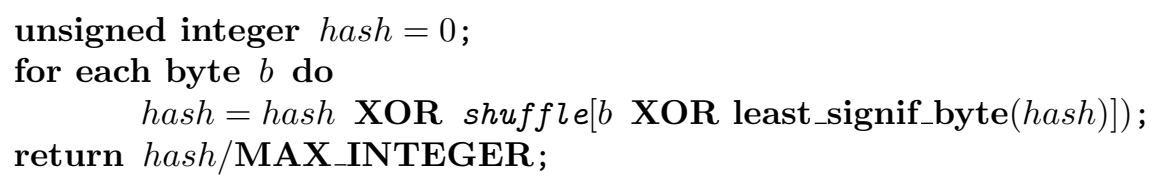

To integrate optimized buffering into an actual epidemic protocol (see Section 2), we have to modify the protocol as follows. Previously, members satisfied the retransmission of a message out of their own buffers. With the optimization, if a member does not have the message buffered locally, it calculates the set of bufferers for the message and picks one at random. The member then sends a retransmission request directly to the bufferer, specifying the message identifier 
and the destination address. A bufferer, on receipt of such a request, determines if it has the message buffered. If so, it satisfies the request. If not, it ignores the request.

Note that processes still have to maintain some information about the messages they have, or have not, received. In the original protocol, processes had to buffer all messages until they are believed to be stable (a global property). In the optimized protocol, processes only need to remember the identifiers of messages they have received locally. They can do so in sorted lists of records, one list per sender. Each record describes, using two sequence numbers, a range of consecutively received messages. Since there are typically not many senders, and each list will typically be of size 1 , the amount of storage required is negligible.

The buffering requirements of the epidemic protocol are improved as follows. In the original protocol, the memory requirement for buffering on each member grew $O(\rho \log n)$, where $\rho$ is the total message rate and $n$ is the number of participants (assuming fixed sized messages and fixed message loss rate) $\mathrm{BHO}^{+} 99$. This is because the number of rounds of gossip required to spread information fully with a certain probability grows $O(\log n)$. In the modified protocol, the buffering requirement on each member shrinks by $O(\rho \log n / n)$, since $C$ is constant.

\section{Improvement}

Up until now we have assumed that the only message loss was due to rare and independent receive omissions. In this section, we will suggest an improved strategy in order to deal with more catastrophic message loss, without sacrificing the advantageous scaling properties. The improvement consists of two parts.

The first part is to maintain two message buffers. The so-called long-term buffer is like before, in which messages are kept for which the corresponding process is a bufferer. The short-term buffer is a buffer in which all messages are kept in FIFO order as they are received for some fixed amount of time. (Since messages are kept for a fixed amount of time, the size of this buffer is linearly dependent on the message rate $\rho$, but independent of group size.) Both buffers can be used for retransmissions.

The second part involves an improvement to the initial multicast phase. The idea is to detect send omissions or large dependent receive omission problems, and retransmit the message by multicasting it again (rather than by point-topoint repairs). Such strategies are already built into multicast protocols such as bimodal multicast $\left[\mathrm{BHO}^{+} 99\right]$ and $\mathrm{SRM}\left[\mathrm{FJL}^{+} 97\right]$. Thus, the initial multicast phase is subdivided into three subphases:

1a. an unreliable multicast attempts to reach as many members as possible;

1b. detection of catastrophic omission;

1c. multicast retransmission if useful.

For example, in a typical epidemic protocol this can be done as follows. Members detect holes in the incoming message stream by inspecting sequence 
numbers. They include information about holes in gossip messages. When a member receives a gossip with information about a hole that it has detected as well, it sends a multicast retransmission request to the sender. The probability of this happening is low in case of a few receive omissions, but high in the case of a catastrophic omission. The sender should still have the message in its short-term buffer to satisfy the retransmission request. Since these retransmission requests are only triggered by randomized gossip messages, it will not lead to implosion problems such as seen in ack or nak based protocols.

These two parts, when combined, lead to two significant improvements. First, they make catastrophic loss unlikely, so that the assumptions of the original basic optimization are approximately satisfied. Secondly, since most message loss is detected quickly, retransmissions will typically be satisfied out of the short-term buffer without the need for retransmission requests to bufferers. The long-term buffer is only necessary for accomplishing all-or-none semantics in rare failure scenarios.

\section{Simulation Results}

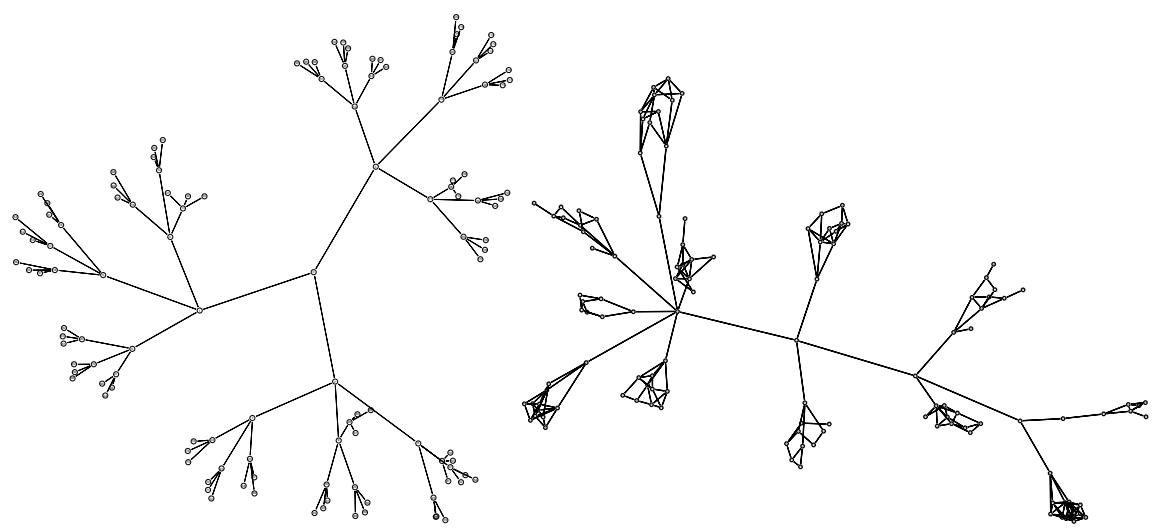

(a)

(b)

Fig. 1. (a) a tree topology; (b) a transit-stub topology.

To validate our techniques, we have simulated bimodal multicast $\mathrm{BHO}^{+} 99$ with and without our buffering optimization. This protocol follows the description of epidemic protocols in Section 2 closely, and contains a multicast retransmission scheme similar to the one described in Section [5. For our simulations, we used the ns2 network simulator $\left[\mathrm{BBE}^{+} 99\right.$, and multicast messages from a single sender. In all experiments, we set $C$ so that $P_{\text {fail }} \approx 0.1 \%$, based on the link loss probability and the number of members (see Equation 3). 
We simulated on two different network topologies (see Figures 1(a) and (b)): a pure tree topology, with the sender located at the root of the tree, and a transit$s t u b$ topology (generated by the ns2 gt-itm topology generator), with the sender located on a central node. The transit-stub topology is more representative of the Internet than is the tree topology. The topology has an influence on the probability of message loss, but as we shall see, the overall effect of these two topologies on the buffering requirements is similar.

In Figures 2 and 3, we show the required amount of buffer space (the maximum number of messages that needed to be buffered) per member as a function of group size. In all these experiments, the individual link-loss probability in the network is $0.1 \%$. In these cases, $C \approx 6$. The graphs for the original bimodal multicast are labeled "pbcast-ipmc," while the buffer optimized multicast graphs are labeled "pbcast-hash." Figure 2 uses a rate of 25 messages/sec. In (a) we used the tree topology, and in (b) the transit-stub topology. Figure 3 shows the same graphs for 100 messages/sec. We find not only that the buffering optimization greatly reduces the memory requirements on the hosts, but also that the buffering behavior is more predictable.

To see the effect of message rate and noise rates more clearly, see Figure 4. In both experiments we used a tree topology with 100 members. In (a), the link-loss probability is still fixed at $0.1 \%$, while the message rate is varied from 25 to 100 messages/sec. In (b), the message rate is fixed at 100 messages/sec, but the link loss probability is varied from $0.1 \%$ to $1.5 \%$. At $P_{l l}=1.5 \%$, we find that $C \approx 9$.

Figure 5 shows that the buffer optimization significantly reduces the memory requirements on each individual host, and also that the buffering responsibility is spread evenly over all members. Again, we are using a tree topology with 100 members. We show how much was buffered on each one of the members using both the original protocol and the optimized one. In (a), the message rate is 25 messages/sec, while in (b) it is 100 messages/sec.

In Figure 6, we show on how many locations each of the messages numbered 1000-1500 was buffered for two different link-loss probabilities: (a) $0.1 \%$ and (b) $1.5 \%$. With larger loss, it is necessary to buffer messages in more locations in order to get the same $P_{\text {fail }}$ probability. Because of this, the probability that nobody buffers a message $\left(1-P_{2}\right)$ is actually smaller for situations with larger loss. The graphs demonstrate this clearly. Note that although, in (a), three messages were not buffered anywhere, this does not imply that the messages were not delivered to every member. In fact, all three messages were correctly delivered.

\section{Related Work}

Work on buffering in group communication can be classified in three categories:

1. Multicast flow control techniques attempt to control the amount of buffering using rate or credit-based mechanisms; 


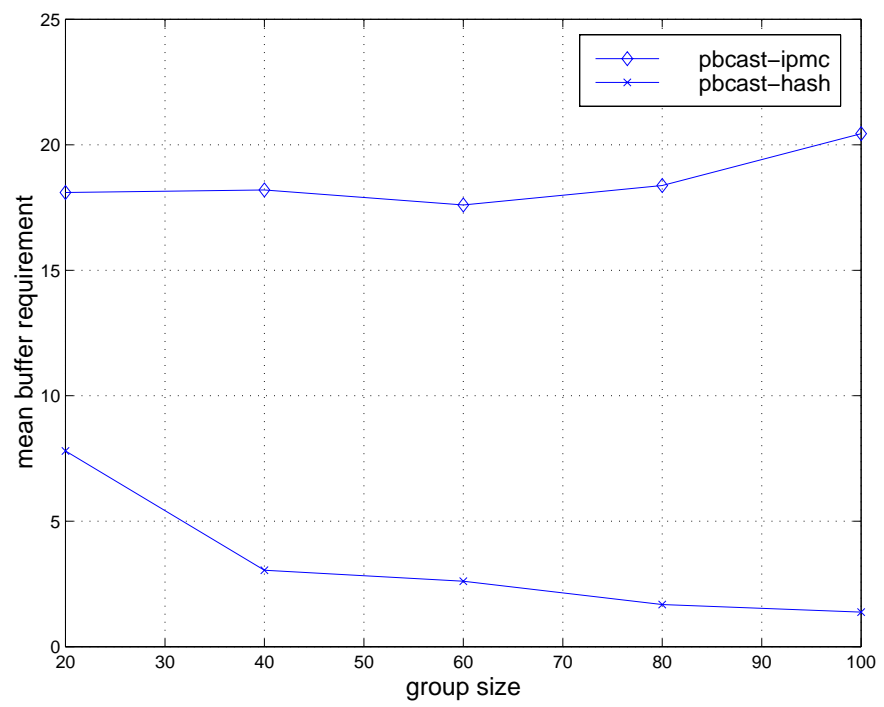

(a)

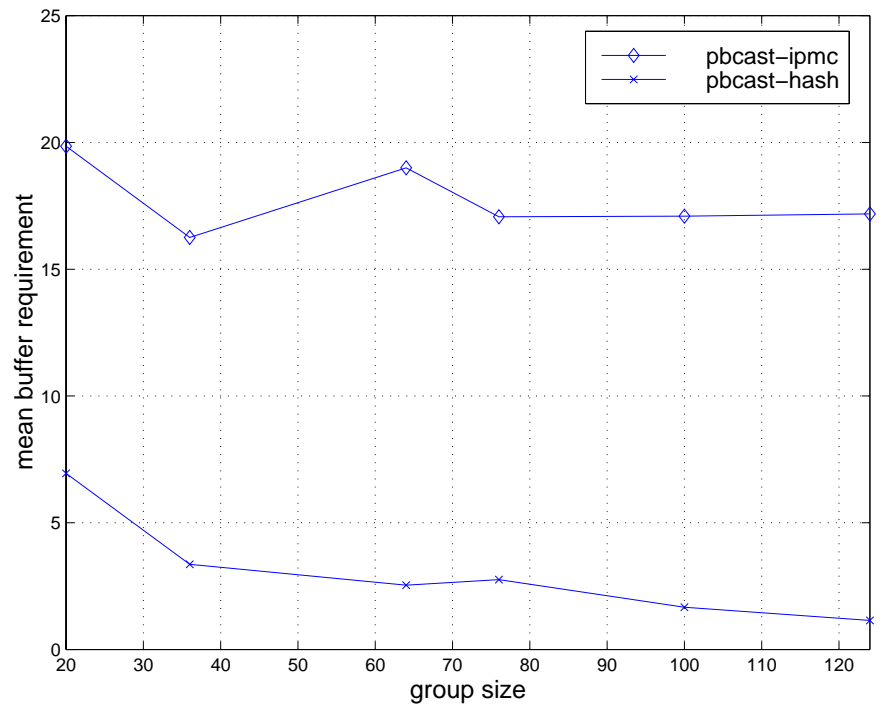

(b)

Fig. 2. The required amount of buffer space per member as a function of group size. In (a) we use a tree topology, while in (b) we use a transit-stub topology. The message rate is 25 messages/sec. 


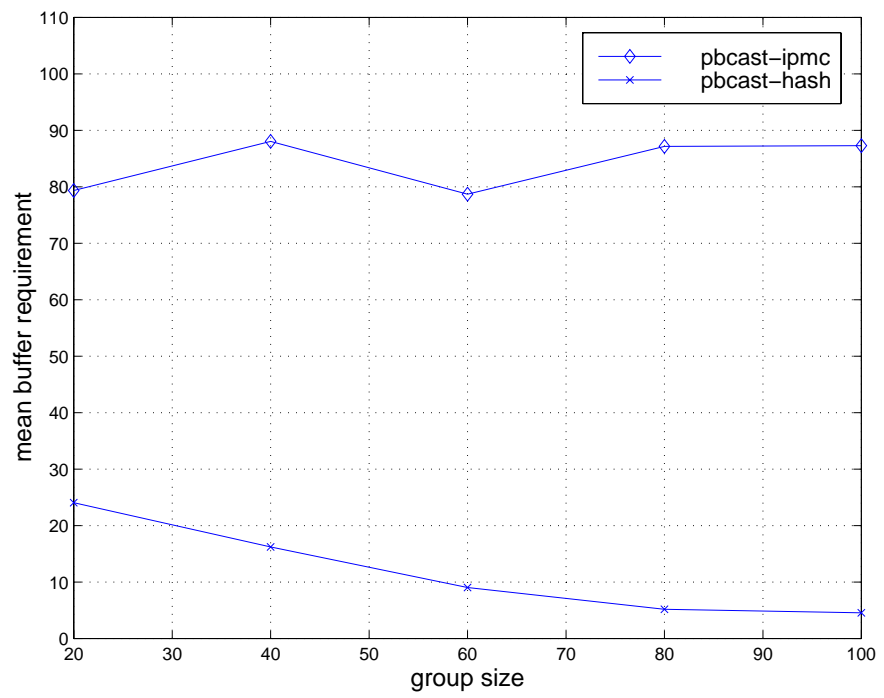

(a)

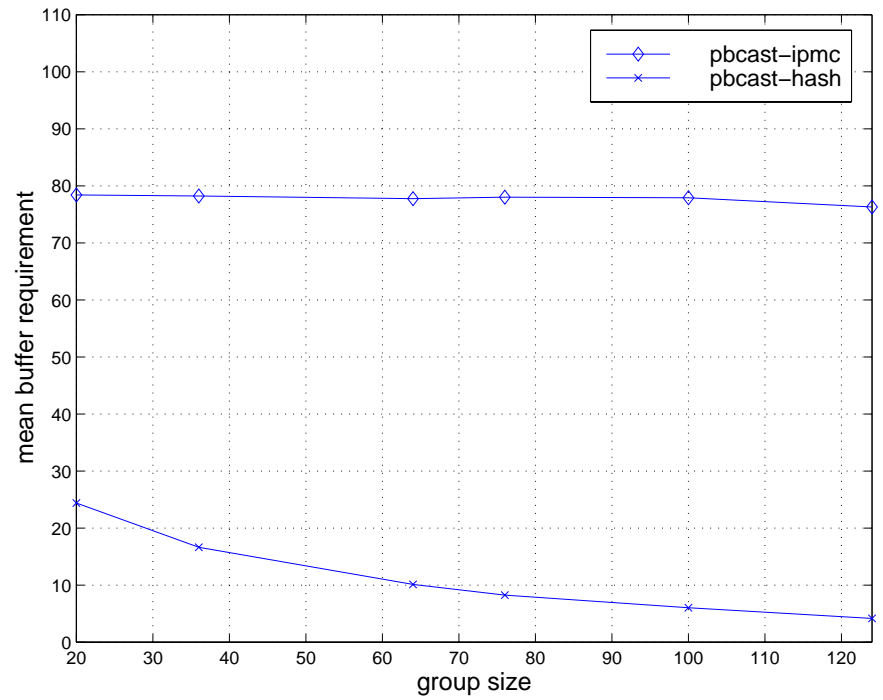

(b)

Fig. 3. Same as Figure 2 but for 100 messages/sec. 


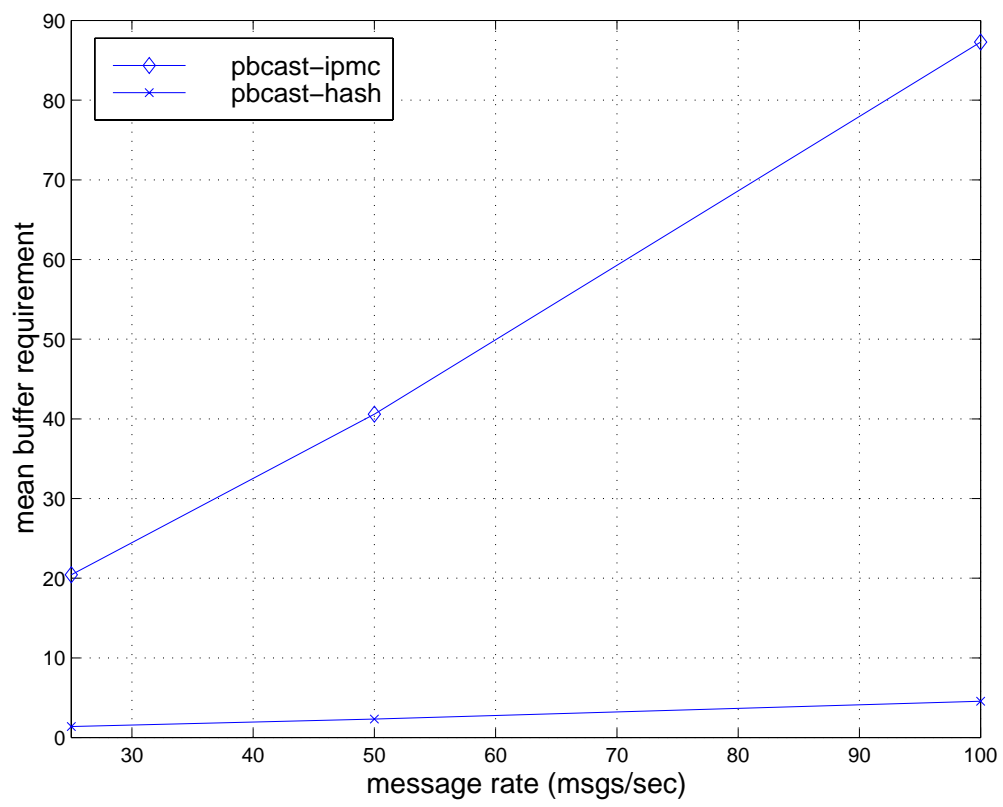

(a)

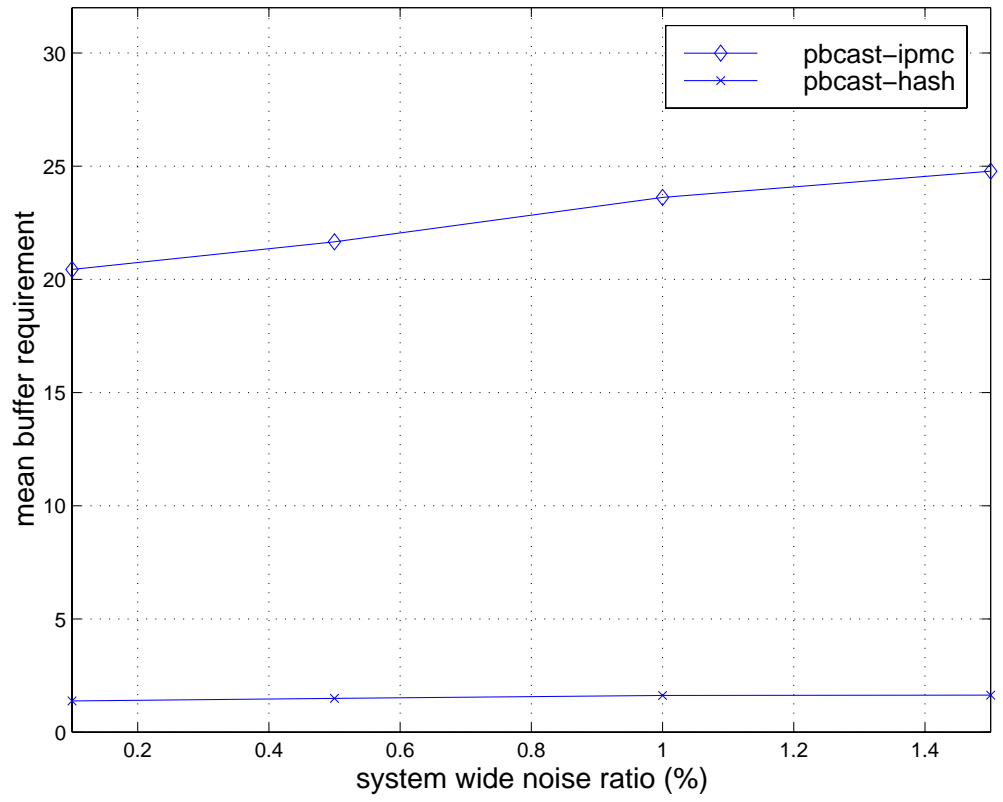

(b)

Fig. 4. In (a), we show the average amount of buffer space per member as a function of message rate. In (b), we show the buffer space as a function of link loss probability. 


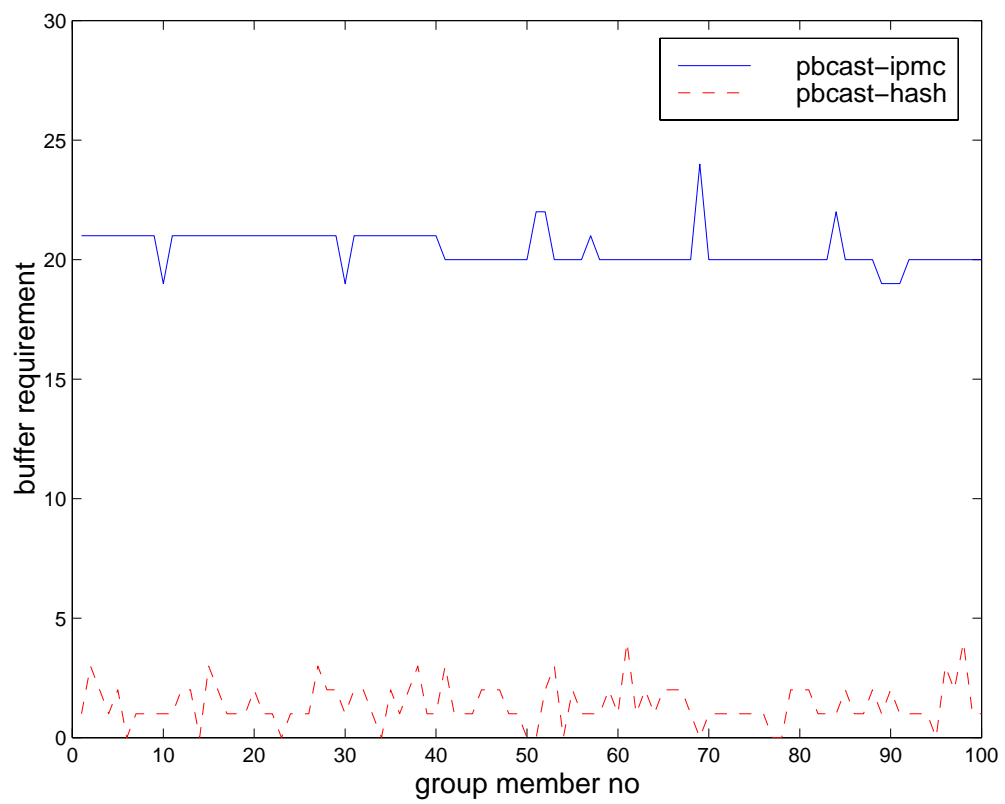

(a)

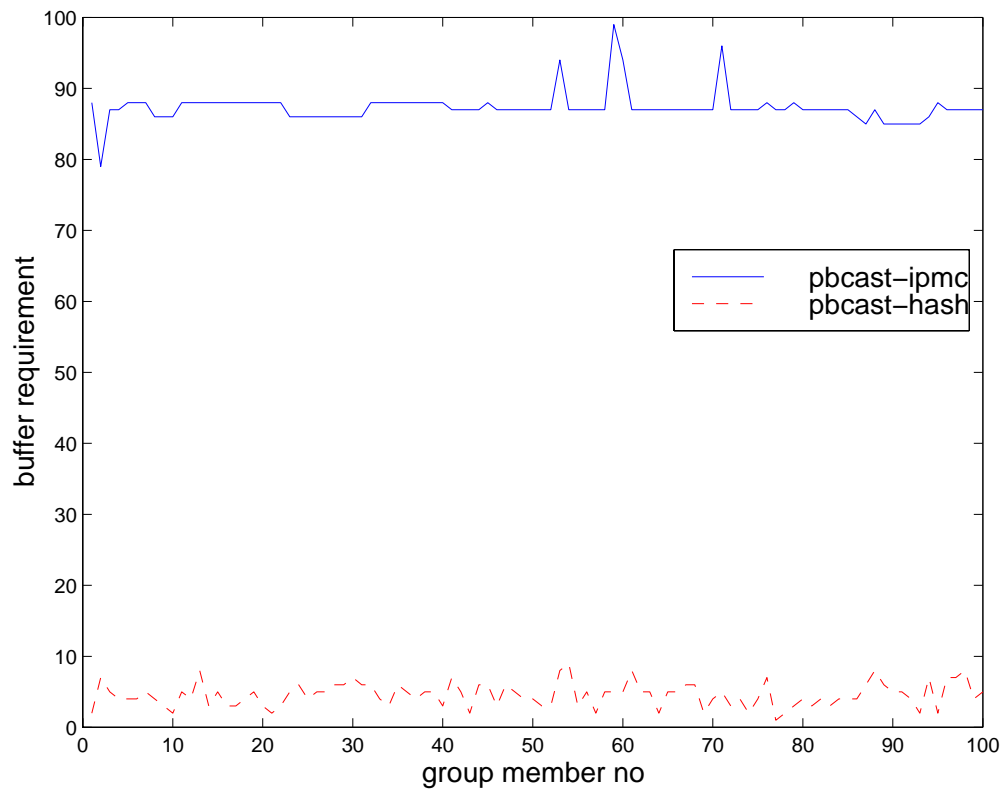

(b)

Fig. 5. This graph shows, for each member, how much buffer space was required. In (a), the message rate is 25 messages/sec, while in (b) the message rate is 100 messages/sec. 


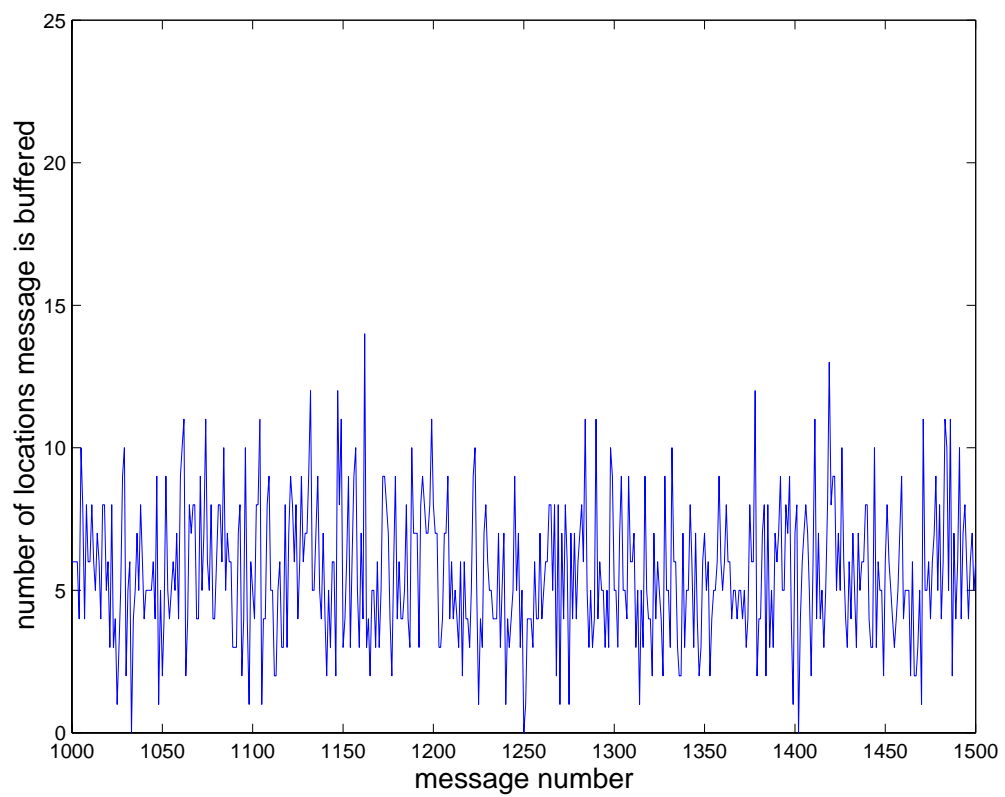

(a)

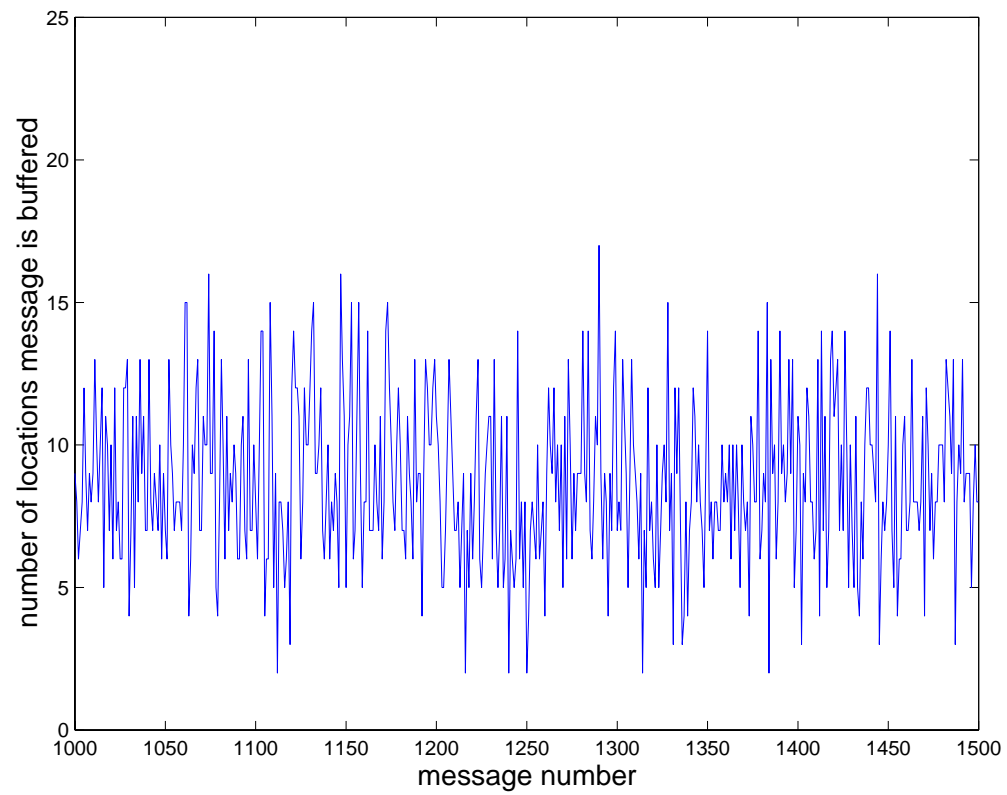

(b)

Fig. 6. This graph shows, for 500 messages starting at message 1000, on how many locations each of these messages was buffered. In (a), the link loss probability is $0.1 \%$, which in (b) this probability is $1.5 \%$. 
2. Stability optimization techniques attempt to minimize the time to achieve and detect stability of messages, thereby reducing the time that messages are buffered;

3. Memory reduction techniques attempt to minimize the amount of buffer memory necessary.

In the first category, a good representative paper is by Mishra and Wu MW98. They study the effect of buffering of rate and credit-based flow control in both ACK and NAK-based multicast protocols using simulation. They conclude that rate-based flow control techniques are generally best. We note that flow control is mostly orthogonal to the buffer optimization. Flow control is an adaptive mechanism, intended to deal with varying availability of resources. These resource include CPU and memory resources on end-hosts and routers, and bandwidth availability on network links. Buffer optimization is not adaptive, but as it reduces the use of memory resources on the end-hosts, it will have an impact on flow control. (Note that although related, congestion control deals with buffering requirements on routers rather than end-hosts, and is therefore not discussed here further.)

In the second category, all reliable communication protocols attempt to optimize the time to achieve stability. Mishra and Kuntur MK99 present a general technique, which they call Newsmonger, to improve the time to detect stability. This is important when the application requires uniform or safe delivery of messages. As a beneficial side-effect, it also reduces the amount of time that messages need to be buffered. The Newsmonger is a token that rotates among the members, and can be applied to any reliable multicast protocol that provides membership agreement of some sort. The technique, when combined with our buffering optimization, is still useful to improve the latency of uniform delivery.

Our buffer optimization technique belongs in the third category. The best known work in this category is a general protocol model called Application Level Framing (ALF) CT90. ALF leaves many reliability decisions to the application, rather than providing an abstraction of a reliable multicast channel. SRM $\left[\mathrm{FJL}^{+}\right.$97 is a well-known implementation of a multicast facility in the ALF model, and is used in various tele-conferencing applications. SRM does not buffer or order messages, but provides call-backs to the application when it detects that a message is lost. It is the application that decides whether and how it wants to retransmit the message. Rather than buffering messages, the application may be able (and, in current SRM applications, usually is able) to regenerate messages based on its state. In contrast to our work, SRM does not provide all-or-none delivery with any known level of reliability.

\section{Conclusion}

In this paper, we presented a technique that significantly optimizes buffer requirements in reliable multicast protocols. In particular, the buffer requirements on a host are reduced by a factor of $n / C$, where $n$ is the size of the group, and $C$ is a 
small constant containing the number of sites where a message should be buffered (typically on the order of about 10). The reliability guarantees of the protocol are slightly adversely affected, but the probability of this can be calculated and limited by choosing a suitable $C$. Using simulation, we have demonstrated that this technique is highly effective.

We have described how buffer optimization can be incorporated into an epidemic multicast protocol such as bimodal multicast [ $\left.\mathrm{BHO}^{+} 99\right]$. In the future, we would like to study the impact of our optimization on other, non-epidemic, reliable multicast protocols. Since such protocols do not allow occasional violations of the all-or-none delivery guarantee, additional mechanisms may be necessary. For example, in so-called virtually synchronous protocols, the conflict can be solved by simulating a partition in the membership if a message cannot be recovered. Since such events should be rare, this may be an acceptable solution, as these protocols already deal with real network partitions.

\section{References}

$\left[\mathrm{BBE}^{+} 99\right]$ S. Bajaj, L. Breslau, D. Estrin, K. Fall, S. Floyd, P. Haldar, M. Handley, A. Helmy, J. Heidemann, P. Huang, S. Kumar, S. McCanne, R. Rejaie, P. Sharma, K. Varadhan, Y. Xu, H. Yu, and Zappala D. Improving simulation for network research. Technical Report 99-702, Univ. of Southern California, March 1999.

$\left[\mathrm{BHO}^{+} 99\right]$ K.P. Birman, M. Hayden, O. Ozkasap, Z. Xiao, M. Budiu, and Y. Minsky. Bimodal multicast. ACM Transactions on Computer Systems, 17(2):41-88, May 1999.

[CT90] D.D. Clark and D.L. Tennenhouse. Architectural considerations for a new generation of protocols. In Proc. of the '90 Symp. on Communications Architectures 85 Protocols, pages 200-208, Philadelphia, PA, September 1990. ACM SIGCOMM.

$\left[\mathrm{DGH}^{+} 87\right]$ A. Demers, D. Greene, C. Hauser, W. Irish, J. Larson, S. Shenker, H. Sturgis, D. Swinehart, and D. Terry. Epidemic algorithms for replicated database maintenance. In Proc. of the Sixth ACM Symp. on Principles of Distributed Computing, pages 1-12, Vancouver, British Columbia, August 1987. ACM SIGOPS-SIGACT.

$\left[\mathrm{FJL}^{+}\right.$97] S. Floyd, V. Jacobson, C.-G. Liu, S. McCanne, and L. Zhang. A reliable multicast framework for light-weight sessions and application level framing. IEEE/ACM Transactions on Networking, 5(6):784-803, December 1997.

[GLW94] R.A. Golding, D.D. Long, and J. Wilkes. The refdbms distributed bibliographic database system. In USENIX Winder 1994 Technical Conference Proceedings, January 1994.

[HT94] V. Hadzilacos and S. Toueg. A modular approach to the specification and implementation of fault-tolerant broadcasts. Technical Report TR94-1425, Department of Computer Science, Cornell University, 1994.

[LP96] J.C. Lin and S. Paul. Rmtp: A reliable multicast transport protocol. In Proc. of IEEE INFOCOM'96, pages 1414-1424, March 1996.

[MK99] S. Mishra and S.M. Kuntur. Improving performance of atomic broadcast protocols using the newsmonger technique. In Proc. of the 7th IFIP International Working Conference on Dependable Computing for Critical Applications, pages 157-176, San Jose, CA, January 1999. 
[MW98] S. Mishra and L. Wu. An evaluation of flow control in group communication. IEEE/ACM Transactions on Networking, 6(5), October 1998.

$\left[\mathrm{PST}^{+} 97\right]$ K. Petersen, M.J. Spreitzer, D.B. Terry, M.M. Theimer, and A.J. Demers. Flexible update propagation for weakly consistent replication. In Proc. of the Sixteenth ACM Symp. on Operating Systems Principles, pages 288-301, Saint-Malo, France, October 1997.

[vRMH98] R. van Renesse, Y. Minsky, and M. Hayden. A gossip-style failure detection service. In Proc. of Middleware'98, pages 55-70. IFIP, September 1998. 